\title{
On Analysis of the Secular Variation
}

\section{A Hydromagnetic Constraint: Theory}

\author{
P.H. ROBERTS \\ Department of Mathematics, University of Newcastle upon Tyne, England
}

S. SCOTT

Rutherford College of Technology, Newcastle upon Tyne, England.

(Received April 25, 1965)

\begin{abstract}
It is noted that the time-scale of the secular variation of the geomagnetic field is rather short compared with the electromagnetic diffusion times appropriate to the earth's core. It is therefore suggested that the secular variation is primarily due to the rearrangement of pre-existing lines of force emanating from the core, and not due to the creation of new (or destruction of old) flux tubes by electromagnetic diffusion. The theoretical consequences of this idea are fully examined.
\end{abstract}

\section{Introduction}

In 1838 Gauss showed in a celebrated paper that, to a high degree of approximation, the main geomagnetic field is a potential field due to sources within the earth. Since his time, as better and more extensive data became available, there have been many repetitions of his analysis in terms of spherical harmonics. One motivation has been a desire to possess maps that exhibit the world-wide structure of the field, and not merely its values at a number of isolated data points. This, however, has not been the only objective. Indeed, if it were, it might have been argued (particularly recently) that, from the standpoint of numerical accuracy, spherical harmonics are not the best choice for the representation series. There has been another* objective: that of understanding the nature of the sources of field. Here spherical harmonics have two important physical advantages. First, they facilitate the separation of the field due to external sources from that due to internal sources. Second, they allow, in principle, the field at the earth's surface $(r=a)$ to be readily extrapolated to the core boundary $(r=b)$ : each term of the representation series is multiplied by $(a / b)^{n}$, where $n$ is its harmonic number**. In practice, the data have proved insufficient in quantity

\footnotetext{
* There have also, of course, been many other objectives, e.g. to be able to compute the trajectories of charged particles in the earth's environment.

** Provided, that is, ferromagnetic sources in the mantle are absent. To the extent that the earth is spherically symmetric, steady currents flowing in the mantle are toroidal and therefore produce no detectable magnetic fields at $r=a$. It is therefore permissible to assume, as we have done here, that a geomagnetostatic potential exists throughout the mantle and which satisfies Laplaces' equation. This conclusion is not correct for the secular variation since unsteady currents in the core can induce toroidal currents in the mantle if conducting. However, this effect may be small, and will be neglected here (see below).
} 
and quality, to permit meaningful extrapolation. More precisely, when the representation series is truncated as late as the data can possibly warrant, it shows no very obvious signs of convergence when extrapolated term by term to the core surface. In principle, other methods of extrapolation are possible. For example, after the analysis of the field into physical sources, such as dipoles, (cf. Alldredge and Hurwitz, 1964a; Lowes and Russell, 1965), the field of each could be computed over the core boundary, and the resulting terms be resummed. Another avenue has been opened by the integral methods of Vestine et al (1963). It seems, however, that, whatever method is employed, severe uncertainties remain in the values calculated at the core surface.

The impracticability of extrapolation appears in a much more acute form when the secular variation field is considered. Here the time scale of the phenomenon so strongly indicates the field is internal in origin that only terms in ascending powers of $(a / r)$ are retained in the harmonic series. Even so, the data itself is of such poor quality that direct extrapolation to the core is virtually meaningless, and it is difficult to believe that further progress is possible without some additional physical postulate. In this paper, we propose the introduction of a very simple result from hydromagnetics; the normal flux of magnetic field from any element on the surface of a body of perfectly conducting fluid remains constant during any motions of the fluid (even though they convect, dilate and distort this element). Or, as it is sometimes restated, the magnetic field is "frozen in" the fluid as it moves. In $\S 2$, we discuss how far this result is applicable to the geomagnetic field and its secular variation. We should observe here that the introduction of a physical hypothesis in the analysis of geomagnetic data is by no means new. For example*, prior to the division of the geomagnetic field into harmonics, it is often (e.g. Vestine et al, 1947; Fougere and Mc Clay, 1957) smoothed in such a way that the line integral of the field round any horizontal curve is zero. In other words, the hypothesis is made that ground to air currents are absent. Now this assumption is not literally and precisely correct, and were data of unlimited accuracy available, this could be demonstrated. But, in making the hypothesis, the belief and claim is that deviations from the hypothesis are so small that they are lost in the general noise, at the present level of data quality. In precisely the same way, we do not regard our hydromagnetic model as being literally and precisely obeyed at the core surface. Rather, we believe that deviations from it are likely to be small or absent in the truncated representation series at the earth's surface. As a corollary it follows that our confidence in the orthodox secular variation analysis is weakened.

The hydromagnetic assumption clearly introduces a link between the main field and its secular variation, and in principle, therefore, its use prohibits an analysis of either separately. However, since the data for the main field are so much more reliable than for its secular

\footnotetext{
* Other examples from geomagnetism are (a) the analyses by Fougere (1964) and Fougere and McClay (1957) of the main field in which the pythagorean relationship $F^{2}=X^{2}+Y^{2}+Z^{2}$ of the $X, Y$ and $Z$ components of total intensity $F$ were used as a constraint, (b) the analysis by Scott (1965a) of the field using the time derivative of the pythagorean relationship $F \dot{F}=X \dot{X}+X \dot{X}+Z \dot{Z}$ as constraint. Other applications of the idea are common in data processing, cf.e.g. "Lectures on numerical weather prediction" by Berkofsky (1962).
} 
variation, it is likely that, when appropriate statistical weights are allotted to allow for this fact, the main field analysis is almost independent of that of the secular variation. The converse is not true, and in this paper we examine the effect of the hydromagnetic hypothesis on the analysis of the secular variation, assuming that the main field is known. It might be objected that we have merely transformed the problem of finding secular variation coefficients to one of finding like coefficients for a harmonic expansion of the horizontal velocity field on the core surface. This is not correct. In principle, given a secular variation field, it is generally not possible to find a velocity field that will generate it from the main field by electromagnetic induction. What our hydromagnetic hypothesis does is to introduce a constraint on the analysis, albeit a constraint of a somewhat unusual nature. It should be noted that, although the explicit connection of main field and secular variation is made at the core surface, the constraint is applied at the earth's surface. Thus our analysis avoids explicit extrapolation of fields to the core, and the concommitant growth of errors. This point is discussed further in $\S 4$.

\section{The Hydromagnetic Assumption}

If the values $\left(\sigma \simeq 3.10^{5} \mathrm{mho} / \mathrm{m}\right)$ often quoted for the conductivity of the core are correct, it is, by laboratory standards, a rather poor conductor. However, from the standpoint of the hydromagnetics of the secular variation, it is an extremely good conductor, as we shall now demonstrate.

The change in magnetic field at a fixed point due to the motion of an electrically conducting fluid is often visualized in two parts. First, there is a change in the field due to the convection of the magnetic flux tubes by the motion. Second, there is a diffusion of the field lines relative to the motions due to ohmic dissipation. The relative importance of these two contributions is gauged by the magnetic Reynolds number, $R_{m}$. This is defined by

$$
R_{m}=\frac{U L}{\eta},
$$

where $L$ is a length characteristic of the magnetic field, $U$ is a velocity characteristic of the flow, and $\eta\left(\mathrm{cm}^{2} / \mathrm{sec}\right)$ is the magnetic diffusivity which, in electromagnetic units, is defined by

$$
\eta=\frac{1}{4 \pi \sigma \mu}
$$

where $\sigma$ is electrical conductivity and $\mu$ is permeability (e.m.u.). When $R_{m} \gg 1$, the change in magnetic field is primarily due to the convection of flux tubes by the motions. This may readily be seen if we re-write the condition $R_{m} \gg 1$ as

$$
\tau_{o} \equiv \frac{L^{2}}{\eta} \gg \frac{L}{U} \equiv \tau_{u}
$$

According to (3) the electromagnetic decay time $\tau_{\sigma}$, during which sources can create new flux tubes and ohmic decay can destory old, is large compared with the dynamical time- 
scale $\tau_{u}$, during which the fluid motions can substantially alter the field by redistributing the lines of force*. If we take $L \approx 10^{8} \mathrm{~cm}$ and $U \approx 1 \mathrm{~mm} / \mathrm{s}$, as characteristic of the secular variation, we find $\tau_{u} \approx 30$ years. But if we accept the estimate $\sigma=3.10^{-6}$ e.m.u. mentioned earlier, and set here (and henceforth) $\mu=1$, we obtain $\eta=2.410^{4} \mathrm{~cm}^{2} / \mathrm{sec}$; whence $\tau_{\sigma} \approx 12,000 \mathrm{yr}$. Thus (3) holds for the secular variation.

It seems to us inevitable, then that the main body of the core may be treated as a perfectly conducting fluid when the secular variation is discussed. Allan and Bullard (1965) have, however, urged that there is a loophole in the argument just given. While not disputing that $\tau_{\sigma}$ governs the rate of separation of field lines from fluid, they point out that this would still imply a large secular change when the field involved is large. Now, it is generally agreed that the toroidal fields, though weak at the surface of the core (because of the high resistivity of the mantle), are large at great depths (because of the shearing of poloidal field by fluid motions). Any sufficiently rapid upwelling of fluid will convect the toroidal field to the core surface and, inevitably, some will separate from the fluid by ohmic diffusion, and will spread into the mantle. To estimate the consequent secular change, Allan and Bullard have constructed models in which an upwelling motion is suddenly "switched on" in a halfspace of fluid which contains a field initially tangential to the (plane rigid) boundary, and increasing in strength linearly with distance from it. Using the equations of electromagnetic induction, they computed the subsequent growth of field in the (insulating) exterior of the fluid. They found that the dominant harmonic generated by the upwelling gives a field of the form

$$
Z=Z_{\infty} f\left(\eta t / L^{2}\right),
$$

where $L$ is a length characteristic of the applied field gradient or of the depth the upwelling reaches, whichever is less. The constant $Z_{\infty}$ is the value attained by $Z$ in the final steady state attained when the diffusion of field through the moving fluid is everywhere equal and opposite to the convective motions; it is proportional to the applied field gradient, the velocity of upwelling, and the conductivity; $f$ approaches 1 monotically as its argument increases. The field normal to the surface is positive on one side of the upwelling, negative on the other, and might be roughly described by a dipole placed beneath the surface and directed antiparallel to the applied field.

The interpretation of Allan and Bullard's model which seems most natural is to suppose $Z_{\infty}$ is of the same order as an anomaly of the main field, say 0.1 gauss at the earth's surface, at most (cf. e.g. Runcorn, 1956). This is compatible with the observation (cf. Runcorn, loc. cit., p. 511) that the lifetime of a centre of secular change is roughly equal to the magnitude of a non-dipole anomaly divided by a typical secular change rate. Quite elementary mag. netohydrodynamic arguments (see boundary layer analysis below) establish that the toroidal

\footnotetext{
* The estimate $L^{2} / \eta$ for $\tau_{\sigma}$ is not, we believe, in conflict with the findings of Chandrasekhar (1946). Chandrasekhar conidered the influence of axisymmetric motions on the decay times for a fluid sphere. He showed that suitably directed motions of sufficient vigour could reduce these times far below $L^{2} / n$, where $L$ is the radius of the sphere. However, we believe this is essentially because these motions convected the regions of strong flux into length scales small compared with $L$.
} 
field gradients are not abnormally large at the core surface, nor is its characteristic scale length small thereat. It is not unreasonable, then, to take $L=500 \mathrm{~km}$ as typical for a large centre of change (cf. Herzenberg and Lowes, 1958). We then obtain (using (4) and assuming $\left.f^{\prime}=0(1)\right)$

$$
\dot{Z} \leq 0\left(\frac{\eta Z_{\infty}}{L^{2}}\right) \sim 3 r / \text { year }
$$

which is rather small to be of interest. Allan and Bullard point out that the initial growth of the function $f$ appearing in (4) is rapid $\left(f^{\prime}(\eta)>1\right.$, small $\left.\eta\right)$. But this is partially a consequence of their model, and if, instead of supposing the velocity is suddenly switched on, we take the more reasonable (though still extreme) view that the body force creating the motion is suddenly switched on, the initial rise of $f$ is more gradual by a factor of $\eta t / L^{2}$ (see Hide and Roberts, 1961, pp. 92-95). Moreover the position is not substantially improved when Allan and Bullard's model is modified by the inclusion of the Navier-Stokes equation to a fully hydromagnetic (perturbation) model; cf. Hide and Roberts, loc. cit.

The value of $\dot{Z}$ obtained in (5) above rests squarely on the hypothesis that $Z_{\infty}$ is comparable in magnitude with an anomaly of the non-dipole field. If one is willing to abandon this idea, it is possible to increase $\dot{Z}$ to reasonable values $(\sim 100 r / y r)$ by increasing $U$ and, therefore, $Z_{\infty}$. But one must then consider the upwelling is shortlived, compared with the lifetime of the centres themselves; otherwise $Z$ would reach values comparable with $Z_{\infty}$. This complication reduces the simplicity and appeal of the model. Moreover, the empirical observation concerning the lifetime of secular variation centres mentioned above is difficult to explain.

One other slight obstacle presented by Allan and Bullard's models should be noted. They would suggest that secular change fields observed at the earth's surface would be best represented by horizontal dipoles positioned beneath the core. However, the opposite view (i.e. vertical dipoles) has been strongly championed by Lowes and Runcorn, 1951.

For all these reasons, we prefer to abandon Allan and Bullard's mechanism in favour of the hydromagnetic assumption; viz., that we may assume $\eta=0$ except, of course, in boundary layers. In the main body of the core, therefore, the equation of electromagnetic induction becomes

$$
\frac{\partial \boldsymbol{B}}{\partial t}=\operatorname{curl}(\boldsymbol{u} \times \boldsymbol{B}),
$$

where $\boldsymbol{u}$ is the fluid velocity. We may note here a simple consequence of (6) which will be useful later. Taking spherical polar coordinates $(r, \theta, \phi)$ based on the geocentre and fixed relative to the mantle, the $r$ component of (4) gives

$$
\begin{aligned}
\frac{\partial B_{r}}{\partial t} & =u_{r}\left[\frac{1}{r \sin \theta} \frac{\partial}{\partial \theta}\left(B_{\theta} \sin \theta\right)+\frac{1}{r \sin \theta} \frac{\partial B_{\phi}}{\partial \phi}\right] \\
& -B_{r}\left[\frac{1}{r \sin \theta} \frac{\partial}{\partial \theta}\left(u_{\theta} \sin \theta\right)+\frac{1}{r \sin \theta} \frac{\partial u_{\phi}}{\partial \phi}\right]
\end{aligned}
$$




$$
+\left(\frac{B_{\theta}}{r} \frac{\partial}{\partial \theta}+\frac{B_{\phi}}{r \sin \theta} \frac{\partial}{\partial \phi}\right) u_{r}-\left(\frac{u_{\theta}}{r} \frac{\partial}{\partial \theta}+\frac{u_{\phi}}{r \sin \theta} \frac{\partial}{\partial \phi}\right) B_{r} .
$$

Equations (6) and (7) hold in the main body of the core but not in a thin boundary layer in contact with the core surface, $\mathscr{S}$. Since we are vitally interested in the behaviour of the field as it leaves the core, it is absolutely essential to consider the structure of the boundary layer in some detail. First we note that the equation

$$
\operatorname{div} \boldsymbol{B}=0
$$

ensures that as we descend from any point of $\mathscr{S}$, assumed spherical, through the boundary layer, $B_{r}$ does not change in the first approximation*, i.e.

$$
\left[B_{r}\right]_{i}{ }^{0}=0 \text {. }
$$

Similarly, since compressibility is unimportant at secular variation frequencies, we have

$$
\left[u_{r}\right]_{i}{ }^{0}=0 .
$$

But at the outer edge $\mathscr{S}_{0}$ of the boundary layer, the fluid is in contact with a rigid solid. Thus

$$
u_{r}\left(\mathscr{S}_{0}\right)=0
$$

From (10) and (11) we have

$$
u_{i}\left(\mathcal{J}_{i}\right)=0 \text {, }
$$

from which it follows, since $\mathscr{S}_{i}$ is spherical, that

$$
\frac{\partial u_{r}}{\partial \theta}=\frac{\partial u_{r}}{\partial \phi}=0 \text { on } \mathscr{S}_{i}
$$

Since $\mathscr{S}_{i}$ is the junction of main stream and boundary layer, (7) also holds thereat. Thus, by (7), (12), (13) we see that on $\mathscr{S}_{i}$

$$
\begin{aligned}
\frac{\partial B_{r}}{\partial t}=- & B_{r}\left[\frac{1}{r \sin \theta} \frac{\partial}{\partial \theta}\left(u_{\theta} \sin \theta\right)+\frac{1}{r \sin \theta} \frac{\partial u_{\phi}}{\partial \phi}\right] \\
- & \left(\frac{u_{\theta}}{r} \frac{\partial}{\partial \theta}+\frac{u_{\phi}}{r \sin \theta} \frac{\partial}{\partial \phi}\right) B_{r} .
\end{aligned}
$$

As a result of (9), we see that (14) holds not only on $\mathscr{S}$ but also on $\mathscr{S}_{0}$, i.e. at the base of the mantle. This is the result we will make use of in $\S 4$. We conclude this section by showing, through further consideration of the boundary layer at $\mathscr{S}$, that not only is the radial component (6), but also the $\theta$ and $\phi$ components of equation (14), are obeyed at $\mathscr{S}_{0}$. This result, in principle, contains information about $\partial \boldsymbol{B} / \partial \mathrm{r}$ on $\mathscr{S}_{i}$, i.e. about the toroidal currents flowing near the core boundary.

\footnotetext{
* The notation $[\ldots]_{i}{ }^{0}$ used in (9) denotes the leap in the quantity...as we pass from the outer edge, $\mathscr{S}_{0}$, of the boundary layer to its inner edge, $\mathscr{S}_{i}$. The reversal of the usual boundary layer terminology has been deemed advisable here, since "inner" and "outer" have obvious meanings in relation to the geocentre. In common with all boundary layer analyses we are in reality considering the leading terms of an asymptotic solution in the limit $n \rightarrow 0$. However, the present "engineering" picture of a layer of finite physical extent has seemed clearer than a strict mathematical description.
} 
The estimate made of $\eta$ made earlier exceeds the values of the kinematic viscosity $\nu\left(10^{-3}\right.$ to $10^{3} \mathrm{~cm}^{2} / \mathrm{sec}$ ) which are often quoted (see, for example, the discussion of Hide and Roberts, 1961). It is likely, then, that the magnetic Prandtl number,

$$
p_{m}=\frac{\nu}{\eta},
$$

is small. And, since $R_{m}$ is large, so is the hydrodynamic Reynolds number

$$
R_{e}=\frac{U L}{\nu} .
$$

Thus, in the bulk of the core, or what in the language of boundary layer theory might be termed 'the main stream,' we may take $\nu=\eta=0$. The consequent lowering of the order of the governing differential equations means that it is impossible, in general, for the main stream to satisfy the no slip conditions on $\boldsymbol{u}$

$$
\boldsymbol{u}=0 \text { on } \mathscr{S}_{0},
$$

and the conditions

$$
\boldsymbol{B} \text { continuous across } \mathscr{S}_{0},
$$

which are required of a physically reasonable solution at the base $\mathscr{S}_{0}$ of the mantle. And, of course, it is the boundary layer which must effect the necessary adjustments.

In the absence of magnetic fields, the neglect neglect of $\nu$ reduces the order of the governing differential equation of hydrodynamics by 2 . Thus the number of boundary demands on the main stream at $\mathscr{S}_{i}$ must be reduced by 2 . It is, in fact, only required, in the first approximations, to satisfy one of the conditions (17), namely $u_{r}=0$, and $\boldsymbol{u}$ is then determined completely. In general, the solution obtained will not satisfy $u_{\theta}=u_{\phi}=0$. This situation is corrected by a boundary layer, of thickness $(\nu \tau)^{1 / 2}$, in which the diffusive term $\nu \nabla^{2}$ is crucial, which matches with the main stream it its inner edge, and which obeys the no slip conditions on $\mathscr{S}_{0}$. (Here $\tau$ denotes the time scale of the variations.)

In the absence of fluid motions, the neglect of $\eta$ reduces the order of the governing differential equations of electrodynamics by 2 . Thus the number of boundary demands on the "main stream" at $\mathscr{S}_{i}$ must also be reduced by 2 . It is, in fact, only required, in the first approximation, to satisfy one of the conditions (18) on $\boldsymbol{B}$, namely that on $B_{r}$, and $\boldsymbol{B}$ is then determined completely. In general, the solution obtained in this way will not satisfy the conditions on $B_{\theta}$ and $B_{\phi}$. This situation is corrected by a boundary layer, of thickness $(\eta \tau)^{1 / 2}$, in which the diffusive term $\eta \nabla^{2}$ is crucial, which matches with the main solution at its inner edge and with all the continuity conditions on $\mathscr{S}_{0}$.

In the presence of both fluid motions and magnetic fields, the approximation $\nu=\eta=0$ reduces the order of the governing differential equations of magnetohydrodynamics by 2 , and not by 4 . In general (more precsely, when $B_{r} \neq 0$ ), the number of boundary demands must be reduced by 2 , and not by 4 . The main stream is, in fact, required, in the first approximation, to satisfy, not only the conditions on $u_{r}$ and $B_{r}$, but also two other conditions. This raises the interesting dilemma, "Do we keep the conditions on $u_{\theta}$ and $u_{\phi}$, or those on $B_{\theta}$ and 
$B_{\phi}$, or do we replace these four conditions by two others?" Stewartson (1957, 1960a, b) first answered this question. He showed that a boundary layer, whose thickness is $(\nu \eta)^{1 / 2} / V_{A}$ (where $V_{A}$ is Alfven velocity based on $B_{r}$; i.e. $V_{A}=B_{r} / \sqrt{ } 4 \pi \mu \rho$ ), exists in which both the diffusive terms $\nu V^{2}$ and $\eta V^{2}$ are crucial. In the first approximation, not only are $u_{r}$ and $B_{r}$ continuous across this layer, but also

$$
\left[u_{\theta, \phi}-\frac{B_{\theta}, \phi}{\sqrt{ } 4 \pi \mu \rho}\left(\frac{\eta}{\nu}\right)^{1 / 2}\right]_{i}^{0}=0
$$

where $\rho$ is the density of the fluid. In other words, if we know the fields $B_{\theta, \phi}\left(\mathscr{S}_{0}\right)$ at the base of the mantle, we must require the main stream solutions to obey

$$
p_{m}{ }^{1 / 2} u_{\theta, \phi}\left(\mathscr{S}_{i}\right)-\frac{1}{\sqrt{ } 4 \pi \mu \rho} B_{\theta, \phi}\left(\mathscr{S}_{i}\right)=-\frac{1}{\sqrt{ } 4 \pi \mu \rho} B_{\theta, \phi}\left(\mathscr{S}_{0}\right)
$$

Only when this has been achieved is it possible to construct a boundary layer which satisfies (17) and (18) at $\mathscr{S}_{0}$.

It is, perhaps, curious that, even though the main stream solutions are based on the assumptions $\nu \rightarrow 0, \eta \rightarrow 0$, a knowledge of their ratio $p_{m}$ is required before they can be determined correctly.

In the geophysical case $\left(p_{m} \ll 1\right)$, $(20)$ reduces simply to

$$
\left[B_{\theta}\right]_{i}^{0}=\left[B_{\phi}\right]_{i}^{0}=0,
$$

i.e. by (9)

$$
[\boldsymbol{B}]_{i}^{0}=0 .
$$

This has three consequences of interest. First, there are no appreciable surface currents in the boundary layer.* Second, the fields we observe at the earth's surface are linked directly to the fields at the edge of the main stream, and are uninfluenced by conditions in the boundary layer. Third, no demands are made of $u_{\theta}$ and $u_{\phi}$ for the main stream at its junction $\mathscr{S}_{i}$ with the boundary layer. It is certainly not to be expected that the no slip conditions will be obeyed.

It may be objected that strictly (19) should only be applied to a steady state. However, as Stewartson (loc. cit.) demonstrated, if condition (19) is violated, an Alfvén wave is emitted by the boundary layer as a kind of tangential shock discontiouity. This passes into the main stream leaving (19) satisfied behind it. In fact, (21) is restored in a time of order $\tau_{A}=\rho / \sigma B_{r}{ }^{2}$

\footnotetext{
* Were the core is solid, the electromagnetic skin-depth $(n \tau)^{1 / 2}$ for a secular variation time scale $\tau$ of 40 years is only $4.10^{6} \mathrm{~cm}$. In many early discussion (e.g. Lowes and Runcorn, 1951, §6) of the secular variation it was erroniously concluded that this result has some bearing on the electrodynamics of the fluid core, and that it therefore followed that the secular variation is produced by intense currents flowing within a depth of $4.10^{6} \mathrm{~cm}$. of $\mathcal{S}$. The fallacy was first pointed out by Roberts $(1954,1955$, 1959; reported by Runcorn, 1954); for further corroboration, see Herzenberg and Lowes (1958). It is curious how, in this geophysical context, allowance for the mobility of the core utterly reverses the conclusions of the skin-depth argument for solid conductors. (Tamao (1959) and Rochester (1960) have recently sought to maintain the earlier conclusion through the analysis of the attenuation of particular Alfvén waves in the presence of Coriolis forces. However, the model chosen is rather special and, in fact, others could equally well be exhibited in which Coriolis forces have no effect at all on the conclusions detailed above.)
} 
$=\eta / V_{A}{ }^{2}$. Thus, if we consider a phenomenon whose time scale $\tau$ is large compard with $\tau_{A}$, the boundary layer can continuously adjust itself so that (21) is always satisfied. In that present geophysical context, $\rho=10 \mathrm{gm} / \mathrm{cc}$, and taking $B_{r}=1 G$, (a conservative estimate), we obtain $\tau_{A}=6$ weeks. Since this is short compared with the secular variations time scale, we may accept (21) without reservation.

As a result of (21) we see that (6) holds not only on $\mathscr{S}$ but also on $\mathscr{S}_{0}$, i.e. at the base of the mantle.

\section{The Nature of the Constraint Imposed by the Hydromagnetic Assumption}

The aim of this section is to discuss the bearing of (14), applied to the main field $\boldsymbol{B}$ and its secular variation $\partial \boldsymbol{B} / \partial t$ at the core surface $r=b$, to the relationship of these fields at the earth's surface $r=a$. The connection can only be established if some physical assumptions concerning the intervening mantle $a>r>b$, are made. The electromagnetic decay time of the mantle has been estimated to be less than four years (cf. Hide and Roberts, 1964, § IV), a time which is rather small compared with that charateristic of the secular variation. It may be concluded that the effects of currents induced in the mantle on the relationship between the field at $r=a$ and that at $r=b$, are fairly small. In this paper, we neglect them entirely, and treat the mantle as an insulator.* The connection between $r=a$ and $r=b$ is then unique (assuming, as is reasonable, no important current sources). To prove this, we may argue as follows:

Since there are no currents outside $r=b$, there exists a geomagnetic potential $V(t)$ such that

$$
\boldsymbol{B}=-\operatorname{grad} V, \quad(r>b),
$$

where

$$
\nabla^{2} V=0
$$

and

$$
V=0\left(r^{-2}\right), \quad r \rightarrow \infty .
$$

By (23), (24) and a well known theorem of potential theory, the specification of $B_{r}(=-\partial V /$ $\partial r$ ) on $\mathscr{S}$ determines $\boldsymbol{B}$ uniquely in $r \geqslant b$.

In principle, from a knowledge of $\boldsymbol{B}$ on the earth's surface $(r=a)$, we may use (22) to (24) to find $B_{r}$ on $\mathscr{S}$. If we know $\boldsymbol{u}$, we may then use (14) to compute $\partial B_{r} / \partial t$ on $\mathscr{S}$, and then use (22) to (24) again to extrapolate the result back to $r=a$, thereby determining $\partial \boldsymbol{B} / \partial t$ on the earth's surface. Even if we do not know $\boldsymbol{u}$, the very postulate that it exists, and satisfies (12), is enough to establish a constraint between $\boldsymbol{B}$ and $\partial \boldsymbol{B} / \partial t$ on $r=a$. It is, in fact, only in

\footnotetext{
* Intuitively, it is clear that the observed time-scale of the secular variation is lengthened by the electromagnetic screening of the mantle. Indeed, it is through a study of this lengthening that the estimate of four years above was reached. For this reason the present authors believe that the neglect of conductivity in the mantle has more serious consequences on their conclusions than, for instance, the possible partial violation of the hydromagnetic assumption discussed in $\S 3$. They hope to return to this question in a later paper.
} 
this sense that we propose to use (14): we postulate no particular form for $\boldsymbol{u}$. The analysis does cast up, as a by-product, some information about $\boldsymbol{u}$, although not enough to determine it uniquely.

It follows from (14) that the flux of $\boldsymbol{B}$ through a surface element of $\mathscr{S}$ as it is convected by $\boldsymbol{u}$ remains constant. It is, as a matter of fact, always possible to define a ficticious "velocity" $\boldsymbol{U}$ whose only purpose is to convect flux tubes. And it might be thought that the existence of such a velocity field contradicts our claim that the hydromagnetic hypothesis is a constraint to our analysis. To examine this point, we observe that, the existence of a vector field $\boldsymbol{U}$ which convects the magnetic field implies

$$
\frac{\partial \boldsymbol{B}}{\partial t}=\operatorname{curl}(\boldsymbol{U} \times \boldsymbol{B})
$$

whence, by Faraday's law of induction

$$
\operatorname{curl} \boldsymbol{E}=-\frac{\partial \boldsymbol{B}}{\partial t}
$$

for the electric field $\boldsymbol{E}$, we have

$$
\operatorname{curl}(\boldsymbol{E}+\boldsymbol{U} \times \boldsymbol{B})=0 .
$$

Thus there exists a single-valued scalar field $\psi$ such that

$$
\boldsymbol{E}+\boldsymbol{U} \times \boldsymbol{B}=-\operatorname{grad} \psi .
$$

It follows from this equation that

$$
\psi=-\int \boldsymbol{E} \cdot \boldsymbol{B} \frac{d s}{B},
$$

where the integral is taken along a line of force. Since there can be no closed lines of force in the exterior of $\mathscr{S}$, (since it is insulating), $\psi$, as defined by (29) is single-valued therein, as demanded above. Now by (28) we have

$$
\boldsymbol{U}=(\boldsymbol{E}+\operatorname{grad} \psi) \times \frac{\boldsymbol{B}}{B^{2}}+\chi \boldsymbol{B},
$$

where $\chi$ is an arbitrary scalar field. If $\chi$ is bounded, the velocity (30) is also bounded everywhere on and outside $\mathscr{S}$, since, by Earnshaw's theorem for a potential field, $\boldsymbol{B}$ can have no zeros in an insulator. It would be possible, if we wished, to choose $\chi$ so that $\operatorname{div} \boldsymbol{U}=0$ outside $\mathscr{S}$; to do this we require that

$$
\chi=-\int \frac{d s}{B} \operatorname{div}\left[(\boldsymbol{E}+\operatorname{grad} \phi) \times \frac{\boldsymbol{B}}{B^{2}}\right],
$$

where the integral is taken along a line of force. However, even if we chose $\chi$ at one of the intersections of the line of force with $\mathscr{S}$ in such a way that $U_{r}=0$, the value at the other intersection, which is then uniquely determined by (31), could not generally be such that $U_{r}=0$. Moreover, even if the condition div $\boldsymbol{U}=0$ is entirely abandoned, it is still not generally possible to make $U_{r}=0$ on $\mathscr{S}$, without making $\chi$, and therefore $\boldsymbol{U}$, infinite thereon. (cf. eq. 
(30); in general, there is no reason why $r \times \boldsymbol{B}$ and $\boldsymbol{E}+\operatorname{grad} \psi$ should be perpendicular at the curves on $\mathscr{S}$ for which $B_{r}=0$.) Thus, our hypothesis of a bounded velocity field $\boldsymbol{u}$ convecting field on is indeed a constraint.

This may also be very easily appreciated in the case in which $\boldsymbol{u}$ is purely toroidal. For then (14) reduces to

$$
\frac{\partial B_{r}}{\partial t}=-\left[\frac{u_{\theta}}{b} \frac{\partial}{\partial \theta}+\frac{u_{\phi}}{b \sin \theta} \frac{\partial}{\partial \phi}\right] B_{r} \text { on } \mathscr{S},
$$

which implies that the foci of the main field on the core surface (i.e. the points of $\mathscr{S}$ at which $B_{r}$ achieves maximum or minimum values) must lie on the curves of zero $B_{r}$. It is also clear that, even when the main field and secular variation fields are compatible so that (32) possesses a solution for $\boldsymbol{u}$, that solution is not unique. For we can add to $\boldsymbol{u}$ any flow round the curves of equal $B_{r}$ drawn on the core surface. (Again, if $B_{r}$ and $\dot{B}_{r}$ are axisymmetric, as they are in some of the examples we have treated, the component of $\boldsymbol{u}$ along lines of longitude is uniquely determined by (14). But is readily seen that any flow round lines of latitude may be added without disturbing the relationship between $B_{r}$ and $\dot{B_{r}}$.)

Some other properties of the hydromagnetic assumption should be noticed. Since the flux of field out of each element of $\mathscr{S}$ is conserved, it follows that the integrated flux, $\mathscr{F}$, out of the core (which, of course, is equal and opposite to the net flux, $-\mathscr{F}$, into the core) is constant in time. This does not mean that any of the multipolar moments are constant in time. Indeed, the axial dipole moment (for example) can take any value between $3 b^{3} \mathscr{F} / 2$ and $-3 b^{3} \mathscr{F} / 2$, the extreme values being taken when the incoming and outgoing lines of force are bunched tightly round each pole. Thus, the theory has no bearing at all on the speculation that the earth's dipole moment is changing (cf. Bullard, 1953).

The fact that $\mathscr{F}$ is conserved on the core surface in no way implies that the net positive (or negative) flux is conserved over the earth's surface. This is made intuitively clear by figure 1 . It can also be demonstrated analytically by a simple example. Suppose the
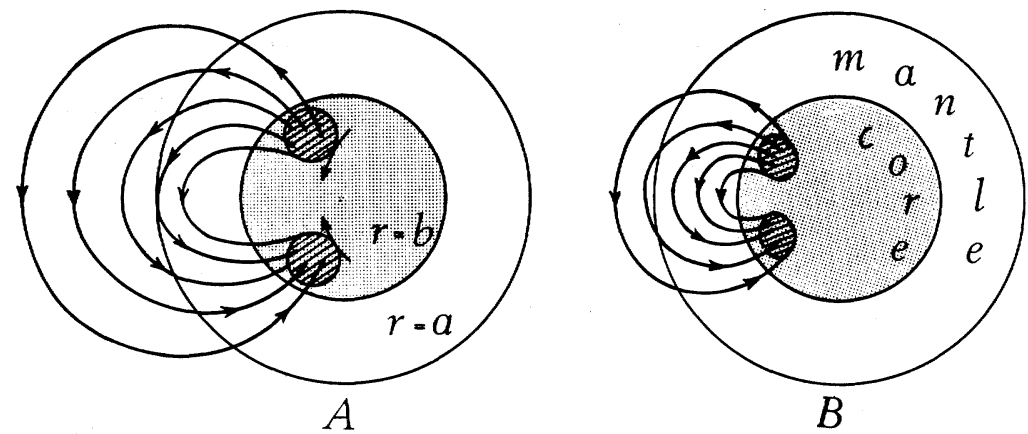

Fig. 1. This schematic diagram compares the disposition of a particular flux tube leaving the core, when the two areas of its intersection with the core surface are moved together by fluid motions (A). It is clear, from the Faraday-Maxwell picture of lines of force, that the tube will shorten and move closer to the core (B). Thus the flux crossing the earth's surface in the outward sense is reduced. The inward flux is, of course, reduced by the same amount. 
magnetic potential $V$ is axisymmetric and given by

$$
V(r, \mu, t)=-k(t)\left[\left(\frac{b}{r}\right)^{2} P_{1}(\mu)+\frac{2}{3} c(t)\left(\frac{b}{r}\right)^{3} P_{2}(\mu)\right],
$$

whence $\mu=\cos \theta, \theta=$ colatitude, $c<1$ and $k$ is chosen to be

$$
k=\frac{27 c^{2} \mathscr{F}}{4 \pi b\left[\left(9 c^{2}-1\right)+\left(1+3 c^{2}\right)^{3 / 2}\right]} .
$$

By (34), the net positive flux over the core surface is $\mathscr{F}$, which we suppose is constant. The net positive flux, $f$, over the earth's surface is then

$$
f=\frac{\mathscr{F}}{b a^{2}}\left[\frac{a\left(9 b^{2} c^{2}-a^{2}\right)+\left(a^{2}+3 b^{2} c^{2}\right)^{3 / 2}}{\left(9 c^{2}-1\right)+\left(1+3 c^{2}\right)^{3 / 2}}\right]
$$

and this certainly varies as $c=c(t)$ varies. (It is also interesting to observe that, if we apply (14) to the field (33), the fluid velocities required to give a secular variation of $200 \mathrm{r} / \mathrm{year}$ at colatitude $60^{\circ}$ from a field of $20,000 \gamma$ are only of the order of $3 \mathrm{~mm} / \mathrm{sec}$, which is not unreasonably large, geophysically.)

It is appropriate here to comment on the relationship of Yukutake's study of the westward drift (Yukutake, 1962) to our analysis. Yukutake introduces a drift velocity $\boldsymbol{v}_{0}{ }^{1}$ of field in the mantle which is, in some senses, analagous to our $\boldsymbol{U}$. Strictly, however, his velocity field $\boldsymbol{v}_{0}{ }^{1}$ cannot convect field lines over the earth's surface unless it is toroidal thereon (compare his eq. (1-4) with eqs. (14) and (32) above). That it should be mainly toroidal on the core surface we have some reason to expect from the westward drift; but that it should also be true on the earth's surface is certainly arguable (cf. Fig. 1 above where an inward component of $\boldsymbol{U}$ would be required on $r=a$ to produce the right-hand diagram from the left.) It should also be noted that the induction equation (14) is linear but not homogeneous in $\boldsymbol{u}$. It is therefore strictly not permissible to superpose solutions obtained from different $\boldsymbol{u}$, as Yukutake has, in essence, done.

\section{Reduction to Matrix Equations}

Let us introduce the customary symbol $Z$ for $-B_{r}$. By (22) to (24) we have

$$
\nabla^{2}(r Z)=0 \text { for } r>b,
$$

where

$$
Z=0\left(r^{-3}\right), r \rightarrow \infty .
$$

Thus, for $r>b$, we may expand $Z$ in a series of the form

$$
Z=\sum_{m=1}^{\infty}\left(\frac{a}{r}\right)^{m+2}\left\{A_{m} P_{m}(\theta)+\sum_{v=1}^{n}\left(A_{m},{ }_{v} \cos v \phi+B_{m}, v \sin v \phi\right) P_{m}^{v}(\theta)\right\} .
$$

We will adopt the convention

$$
A_{m},{ }_{v}=B_{m},{ }_{v}=0 \text {, if } v \leq 0 .
$$

In virtue of (12) we may express $\boldsymbol{u}$ on $\mathscr{S}$ by 


$$
\boldsymbol{u}=\left(0, u_{\theta}(\theta, \phi), u_{\phi}(\theta, \phi)\right) .
$$

However, it does not follow from (12) that $\partial u_{r} / \partial r$ vanishes on $\mathscr{S}$; indeed, over an upwelling (cf. e.g. Coulomb, 1955) this quantity will be large in magnitude. We find it is convenient to separate $\boldsymbol{u}$ (assumed solenoidal near $\mathscr{S}$ ) into a toroidal part $(T$ ), and a poloidal part $(S)$ :

$$
\boldsymbol{u}=\left(0, \frac{\partial S}{\partial \theta}+\frac{1}{\sin \theta} \frac{\partial T}{\partial \phi}, \frac{1}{\sin \theta} \frac{\partial S}{\partial \phi}-\frac{\partial T}{\partial \theta}\right)
$$

Then the scalar $L^{2} S$ takes the value of $b \partial u_{r} / \partial r_{r}$ on $\mathscr{S}$. Here $L^{2}$ is the operator defined by

$$
L^{2}=-\left[\frac{1}{\sin \theta} \frac{\partial}{\partial \theta}\left(\sin \theta \frac{\partial}{\partial \theta}+\frac{1}{\sin ^{2} \theta} \frac{\partial^{2}}{\partial \phi^{2}}\right] .\right.
$$

Using (41), equation (14) may be written as

$$
b \frac{\partial Z}{\partial t}=Z L^{2} S-\left(\frac{\partial S}{\partial \theta} \frac{\partial Z}{\partial \theta}+\frac{1}{\sin ^{2} \theta} \frac{\partial S}{\partial \phi} \frac{\partial Z}{\partial \phi}\right)+\frac{1}{\sin \theta}\left(\frac{\partial T}{\partial \theta} \frac{\partial Z}{\partial \phi}-\frac{\partial T}{\partial \phi} \frac{\partial Z}{\partial \theta}\right) .
$$

Let us expand $T$ and $S$ in harmonic series

$$
\begin{gathered}
T=\sum_{n=1}^{\infty}\left[C_{n} P_{n}(\theta)+\sum_{w=1}^{n}\left(C_{n},{ }_{w} \cos w \phi+D_{n}, w \sin w \phi\right) P_{n}{ }^{w}(\theta)\right], \\
S=\sum_{n=1}^{\infty}\left[E_{n} P_{n}(\theta)+\sum_{w=1}^{n}\left(E_{n},{ }_{w} \cos w \phi+F_{n},{ }_{w} \sin w \phi\right) P_{n}{ }^{w}(\theta)\right] .
\end{gathered}
$$

Multiplying equation (43) by $2 P_{l}^{u}(\theta) \cos u \phi \sin \theta d \theta d \phi / w$ and integrating over all permissible $\theta$ and $\phi$, we obtain, after integration by parts, (adopting Schmidt's normalization of Legendre functions)

$$
\begin{aligned}
& \frac{8 b}{(2 l+1)} \frac{d A_{l}, u}{d t}=\sum_{m=1}^{\infty} \sum_{n=1}^{\infty}\left(\frac{a}{b}\right)^{m-l}\left\{\delta_{u}{ }^{0} A_{m}, 0 E_{n}, 0 \int_{-1}^{1}\left(1-\mu^{2}\right) \frac{d P_{l}^{0}}{d \mu} \frac{d P_{n}{ }^{0}}{d \mu} P_{m}{ }^{0} d \mu\right. \\
& +\sum_{w=0}^{n}\left[\left(A_{m, u+w} D_{n, w}-B_{m}, u+w C_{n}, w\right) \int_{-1}^{1}\left(u P_{l}^{u} \frac{d P_{n}{ }^{w}}{d \mu}-w P_{n}{ }^{w} \frac{d P_{l}^{u}}{d \mu}\right) P_{m}{ }^{u+w} d \mu\right. \\
& +\left(A_{m, u+w} E_{n}, w+B_{m, u+w} F_{n}, w\right) \int_{-1}^{1}\left(\left(1-\mu^{2}\right) \frac{d P_{l}^{u}}{d \mu} \frac{d P_{n}{ }^{w}}{d \mu}-\frac{u w}{1-\mu^{2}} P_{l}^{u} P_{n}{ }^{w}\right) P_{m}{ }^{u+w} d \mu \\
& -\left(A_{m, u-w} D_{n, w}+B_{m, u-w} C_{n}, w\right) \cdot \int_{-1}^{1}\left(u P_{l}^{u} \frac{d P_{n}^{w}}{d \mu}+w P_{n}{ }^{w} \frac{d P_{l}^{u}}{d \mu}\right) P_{m}{ }^{u-w} d \mu \\
& +\left(A_{m}, u-w E_{n}, w_{w}-B_{m}, u-w F_{n}, w\right) \int_{-1}^{1}\left(\left(1-\mu^{2}\right) \frac{d P_{l}^{u}}{d \mu} \frac{d P_{n}^{w}}{d \mu}+\frac{u w}{1-\mu^{2}} P_{l}^{u} P_{n}^{w}\right) P_{m}^{u-w} d \mu \\
& -\left(A_{m, w-u} D_{n, w}-B_{m, w-u} C_{n}, w\right) \int_{-1}^{1}\left(u P_{l}^{u} \frac{d P_{n}^{w}}{d \mu}+w P_{n}{ }^{w} \frac{d P_{l}^{u}}{d \mu}\right) P_{m}{ }^{w-u} d \mu \\
& +\left(A_{m, w-u} E_{n}, w+B_{m, w-u} F_{n}, w\right) \int_{-1}^{1}\left(\left(1-\mu^{2}\right) \frac{d P_{l}^{u}}{d \mu} \frac{d P_{n}{ }^{w}}{d \mu}\right. \\
& \left.\left.\left.+\frac{u w}{1-\mu^{2}} P_{l}^{u} P_{n}^{u}\right) P_{m}^{w-u} d \mu\right]\right\} \text {. }
\end{aligned}
$$

Similarly, by multiplication by $2 P_{l}^{u}(\theta) \sin u \phi \sin \theta d \theta d \phi / \pi$ and integration, we obtain 


$$
\begin{aligned}
& \frac{8 b}{(2 l+1)} \frac{d B_{l, u}}{d t}=\sum_{m=1}^{\infty} \sum_{n=1}^{\infty} \sum_{w=0}^{n}\left(\frac{a}{b}\right)^{m-l}\left\{\left(A_{m}, u+w, C_{n}, w+B_{m}, u+w D_{n}, w\right) \int_{-1}^{1} u P_{l}^{u} \frac{d P_{n}{ }^{w}}{d \mu}\right. \\
& \left.-w P_{n}{ }^{w} \frac{d P_{l}^{u}}{d \mu}\right) d \mu \\
& -\left(A_{m, u+w} F_{n, w}-B_{m}, u+w E_{n}, w\right) \int_{-1}^{1}\left(\left(1-\mu^{2}\right) \frac{d P_{n}^{w}}{d \mu} \frac{d P_{l}^{u}}{d \mu}-\frac{u w}{1-\mu^{2}} P_{l}^{u} P_{n}^{w}\right) . P_{m}^{u+w} d \mu \\
& +\left(A_{m, u-w} C_{n, w}-B_{m, u-w} D_{n}, w\right) \int_{-1}^{1}\left(u P_{l}^{u} \frac{d P_{n}{ }^{w}}{d \mu}+w P_{n}{ }^{w} \frac{d P_{l}^{u}}{d \mu}\right) P_{m}{ }^{u-w} d \mu \\
& +\left(A_{m, u-w} F_{n, w}+B_{m, u-w} E_{n}, w\right) \int_{-1}^{1}\left(\left(1-\mu^{2}\right) \frac{d P_{n}^{w}}{d \mu} \frac{d P_{l}^{u}}{d \mu}+\frac{u w}{1-\mu^{2}}\right) P_{m}^{u-w} d \mu \\
& +\left(A_{m, w-u} C_{n}, w+B_{m}, w-u D_{n}, w\right) \int_{-1}^{1}\left(u P_{l}^{u} \frac{d P_{n}^{w}}{d \mu}+w P_{n}{ }^{w} \frac{d P_{l}^{u}}{d \mu}\right) P_{m}^{w-u} d \mu \\
& \left.+\left(A_{m}, w-u F_{n}, w-B_{m}, w-u E_{n}, w\right) \int_{-1}^{1}\left(\left(1-\mu^{2}\right) \frac{d P_{n}^{w}}{d \mu} \frac{d P_{l}^{u}}{d \mu}+\frac{u w}{1-\mu^{2}} P_{l}^{u} P_{n}^{w}\right) P_{m}^{w-u} d \mu\right\} .
\end{aligned}
$$

The factor $(a / b)^{m-l}$ in (46) and (47) arises from the fact that (43) is applied on $\mathscr{S}$, and that (38) and its time derivative must therefore be evaluated at $r=b$. It is the presence of this factor which precludes the introduction of a non singular velocity $\boldsymbol{U}$ directly onto $r=a$. A large number of the "coupling integrals" appearing on the right of (46) and (47) have been evaluated (Scott, 1965 b).

Let us introduce column vectors of the form

$$
\mathscr{z}=\left(\begin{array}{l}
A_{l}, u \\
B_{l}, u
\end{array}\right), \quad \mathscr{U}=\left(\begin{array}{l}
C_{n, w} \\
D_{n, w}, w \\
E_{n, w} \\
F_{n, w}
\end{array}\right),
$$

(listing $A_{l}$ as $A_{l}, o$ etc). Then equations (46) and (47) may be written as

$$
\sum_{n, w} \mathscr{C}_{(l, u)(n, w)} \mathscr{U}_{(n, w)}=\dot{\mathscr{x}}_{(l, u)},
$$

where $\mathscr{C}$ denotes "the coupling matrix". This matrix is linear in $\mathscr{Z}$. Since the hydromagnetic approximation is a constraint, the matrix $\mathscr{C}$ is singular for all $\mathscr{Z}$; solutions to (49) are only possible when the (secular variation) column vector $\dot{\delta}$ on the right is compatible with $\mathscr{C}$, and the solution then obtained for the velocity coefficients is not then unique.

The structure of (49) will be considered in more detail in the second paper of this series. We offer here only a few remarks. The coupling integrals appearing in (46) and (47) vanish unless the so-called triangle inequalities are satisfied (cf. e.g. Bullard and Gellman, 1954). This has the effect of limiting the exponent of $a / b$ in the $\mathscr{C}_{(l, u)(n, w)}$ element of the coupling matrix by the value $n$. Remembering that $a / b \approx 2$, this has two important effects on (49). First, since $\mathscr{\mathscr { Z }}$ is not dominated by high harmonics (despite the large size of the $\mathscr{C}$ entries having large $n$ ), we must conclude that the high harmonics of $\mathscr{U}$ are small,*i.e. the series

* We are here excluding from the discussion the singular part of $\mathscr{U}$ i.e. the part obtained by solving (49) for $\dot{z}=0$. This singular part could clearly have any magnitude, and we would not know about it at the earth's surface. But this seems rather unlikely. 
we obtain for $\boldsymbol{u}$ shculd be rapidly convergent. Second, small changes in $\not{z}$ generate large changes in the elements of $\mathscr{C}$ of large $n$, and will therefore affect its compatibility properties significantly, but the corresponding changes in $\mathscr{U}$ will be small. In other words, $\boldsymbol{u}$ is insensitive to appreciable (compatible) changes in $\mathscr{Z}$. We return to these matters in the second paper of this series.

Brief reports of this work has already appeared (Yerkes Observatory report in Astrophysical Journal for 1964), and in a thesis (Scott, 1964). We should thank Dr. Vestine for his interest in our ideas when one of us (P.H.R.) explained them to him at the Conference on geophysical data handling held at Yorktown Heights, N.Y. in October 1962.

\section{References}

Allan, D.W. and Bullard, E.C. Rev. Mod. Phys. 30, 1087. 1958.

Allan, D.W. and Bullard, E.C. Unpublished. 1965.

Alldredge, L.R. and Hurwitz, L. J. Geophys. Res. 69, 2631. 1964.

Berkofsky, L. Report AFCRL-62-684. 1962.

Bullard, E.C. 1953. J. Geophys. Res. 58, 277.

Bullard, E.C. and Gellman, H. Phil. Trans. Roy. Soc. Lond. A, 247, 213. 1954.

Chandrasekhar, S. Astrophys. J. 124, 232-265. 1956.

Coulomb, J. Ann. Geophys, 11, 80. 1955.

Fougere, P.F. J. Geophys. Res. 69, 2641. 1964.

Fougere, P.F. and McClay, J.F. Geophys. Res. Paper 55. AFCRC, ASTIA document ADII7268 1957.

Hide, R. and Roberts, P.H. Chapter 1 of "Physics and Chemistry of the Earth," Vol. 4. Pergamon. 1961.

Lowes, F.J. and Herzenberg, A. Ann. Geophys. 14, 526. 1958.

Lowes, F.J. and Runcorn, S.K. Phil. Trans. Roy. Soc. Lond. A, 243, 525. 1951.

Lowes, F.J. and Russell, L.M. to apear. 1965.

Roberts, P.H. unpublished Ph. D. Dissertation, Cambridge. 1954.

Roberts, P.H. Astrophys. J. 122, 315. 1955.

Roberts, P.H. Ann. Geophys. 15, 75. 1959.

Rochester, M.G. Phil. Trans. Roy. Soc. London. A, 252, 431. 1960.

Runcorn, S.K. Handbuch der Physik, 47, 498. 1956.

Scott, S. unpublished dissertation, Newcastle University. 1964.

Scott, S. to appear. 1965a.

Scott, S. to appear. 1965b.

Stewartson, K. Proc. Camb. Phil. Soc. 53, 774. 1957.

Stewartson, K. J. Fl. Mech. 8, 82. 1960a.

Stewartson, K. Rev. Mod. Phys. 32, 855. 1960b.

Tamao, T. Sc. Rep. Tâhuko Univ. (Ser. 5), Geophysics, 10, 137. 1959.

Vestine, E.H. et al. Carnegie Institute Rublications, Washington, No.578. 1947.

Vestine, E.H. et al, J. Geomag. and Geoelec. 15, 47-89. 1963.

Yukutake, T. Bull. Earthquake Res. Inst. 40, 1. 1962. 